\section{Tel2 mediates activation and localization of ATM/Tel1 kinase to a double-strand break}

\author{
Carol M. Anderson, ${ }^{1}$ Dmitry Korkin ${ }^{2,3,4,5}$ \\ Dana L. Smith, ${ }^{1}$ Svetlana Makovets, ${ }^{1}$ \\ Jeffrey J. Seidel, ${ }^{1}$ Andrej Sali, ${ }^{2,3,4}$ and \\ Elizabeth H. Blackburn ${ }^{1,6}$
}

\begin{abstract}
${ }^{1}$ Department of Biochemistry, University of California, San Francisco, California 94143, USA; ${ }^{2}$ Department of Biopharmaceutical Sciences, University of California, San Francisco, California 94143, USA; ${ }^{3}$ Department of Pharmaceutical Chemistry, University of California, San Francisco, California 94143, USA; ${ }^{4}$ California Institute for Quantitative Biomedical Research, University of California, San Francisco, California 94143, USA
\end{abstract}

\begin{abstract}
The kinases ATM and ATR (Tel1 and Mec1 in the yeast Saccharomyces cerevisiae) control the response to DNA damage. We report that $S$. cerevisiae Tel2 acts at an early step of the TEL1/ATM pathway of DNA damage signaling. We show that Tel1 and Tel2 interact, and that even when Tel1 protein levels are high, this interaction is specifically required for Tel1 localization to a DNA break and its activation of downstream targets. Computational analysis revealed structural homology between Tel2 and Ddc2 (ATRIP in vertebrates), a partner of Mec1, suggesting a common structural principle used by partners of phoshoinositide 3-kinase-like kinases.
\end{abstract}

Supplemental material is available at http://www.genesdev.org.

Received December 21, 2007; revised version accepted February 11, 2008.

To guard against loss or alteration of genetic information, all cells have mechanisms for recognizing and repairing DNA damage. The DNA damage response slows or halts cell cycle progression to allow time for repair and up-regulates expression of repair machinery. Defects in this response lead to accumulation of mutations, genomic instability, and, in higher organisms, cancer.

The phoshoinositide 3-kinase-like kinases (PIKKs) ATM and ATR control the response to DNA damage (Abraham 2001). These proteins share structural homology and perform partially redundant roles. ATM (Tel1 in Saccharomyces cerevisiae) responds mainly to doublestrand breaks (DSBs), whereas ATR (S. cerevisiae Mec1) is activated in response to ssDNA (Abraham 2001). ATR/ Mec1 constitutively associates with ATRIP (Ddc2 in $S$.

[Keywords: DNA damage signaling; checkpoint; Clk-2; PI3 kinase-like kinase; $\alpha$-superhelical protein; telomere]

${ }^{5}$ Present address: Informatics Institute and Department of Computer Science, University of Missouri, Columbia, MO 65211, USA. ${ }^{6}$ Corresponding author.

E-MAIL elizabeth.blackburn@ucsf.edu; FAX (415) 514-2913.

Article published online ahead of print. Article and publication date are online at http://www.genesdev.org/cgi/doi/10.1101/gad.1646208. cerevisiae); the ATR-ATRIP complex binds to regions of exposed ssDNA via an interaction with the singlestranded binding protein RPA (Rouse and Jackson 2002; Zou and Elledge 2003). In contrast, ATM/Tell localizes to DSBs via an interaction with the Mre11-Rad50-Nbs1 complex (Mre11-Rad50-Xrs2 in S. cerevisiae, called MRX) (Nakada et al. 2003a; Falck et al. 2005; You et al. 2005).

Tel2 is an essential gene conserved throughout eukaryotes. Evidence from various organisms has implicated Tel2 in the response to DNA damage (Ahmed et al. 2001; Collis et al. 2007; Shikata et al. 2007; Takai et al. 2007). In Caenorhabditis elegans, two mutant alleles of TEL2, rad-5(mn159) and clk-2(qm37), each cause sensitivity to DNA damaging agents and defects in the DNA damage checkpoint and the S-phase replication checkpoint (Hartman and Herman 1982; Gartner et al. 2000; Ahmed et al. 2001). The radiation sensitivity of clk-2 mutants is enhanced by mutations in mrt-2 or hus-1, components of the 9-1-1 complex (Ahmed et al. 2001), suggesting that Clk-2 acts independently of this complex. Furthermore, wild-type Clk-2 is not required for recruitment of ATL-1, the C. elegans ortholog of ATR/ Mec1, to stalled replication forks (Garcia-Muse and Boulton 2005). A study of human cells found a physical association between Tel2 and ATR, ATRIP, and Chk1, although ATR activation and recruitment to sites of damage were not significantly affected by Tel 2 depletion (Collis et al. 2007). The Schizosaccharomyces pombe Tel2 ortholog is required for the response to replication stress (Shikata et al. 2007). Repression of $t e 12^{+}$expression abrogated phosphorylation of Mrc1 and Cds1 (S. cerevisiae Rad53) after treatment with hydroxyurea (HU), indicating that Tel2 functions upstream of Mrc1 and Cds1 in the response to replication stress. However, the exact function of Tel2 has remained unknown.

Here, we report that $S$. cerevisiae Tel2 functions at a specific step in the ATM/Tell pathway in the response to DNA damage. Analyses of damage sensitivity, cell cycle progression after DNA damage, and phosphorylation of key proteins of the DNA damage signaling network together showed that Tel2 is an upstream component of the TEL1 signaling pathway. We demonstrate that Tel1 and Tel2 interact, and that the tel2-1 mutation completely disrupts the Tel1-Tel2 interaction and interferes with localization of Tell to an induced DSB in vivo. While loss of the Tel1-Tel2 interaction modestly decreases the total amount of Tell protein in cells, we demonstrate that the loss of Tell function caused by the tel2-1 mutation is not a result of lower protein levels of either Tel2 or Tel1. Computational analysis showed structural similarity of Tel2 to Ddc2 (ATRIP in vertebrates), a binding partner of Mec1 required for recruitment of Mecl to sites of DNA damage. We show that like Ddc2, Tel2 interacts with an $\alpha$-superhelical region in a portion of Tel1 $\mathrm{N}$-terminal to the kinase domain. These findings reveal that the interaction of $\alpha$-superhelical modules is general strategy used by the PIKKs to interact with their partner proteins.

\section{Results and Discussion}

Because TEL2 orthologs in other organisms play roles in the DNA damage and replication checkpoints, we first determined whether the essential $S$. cerevisiae Tel2 pro- 
tein also affects DNA damage signaling. For these experiments, we used the tel2-1 allele, which encodes the single amino acid change S129N. This mutation causes telomere shortening and mild temperature sensitivity, but cell growth is otherwise apparently normal (Runge and Zakian 1996). In plate growth assays, the tel2-1 mutation alone did not confer damage sensitivity (Fig. 1A; Supplemental Fig. S1), but when combined with a deletion of MEC1, cells grew more slowly on normal medium and were more sensitive to DNA-damaging agents than either single mutant (Fig. 1A). This is reminiscent of the previously reported damage sensitivity of tel1s strains, which, similarly, is uncovered in a mec1s background (Fig. 1A; Morrow et al. 1995). In contrast, tel1s tel2-1 cells showed no damage sensitivity. Notably, the phenotypes of the double mutants mec1s tel2-1 and mec1s tel1s, and the triple mutant mec1s tel1s tel2-1, were all indistinguishable from each other (Fig. 1A; Supplemental Fig. S1B). These data therefore provided evidence that TEL2 acts in the TEL1 pathway of DNA damage signaling.

Either Mec1 or Tell can initiate activation of Rad53, a central transducer of DNA damage signaling (Fig. 1C; Sanchez et al. 1996). The tel2-1 mutation alone caused a delay in Rad53 phosphorylation after treatment with DNA-damaging agents (Fig. 1B). This delay occurred when damage was inflicted in either $\mathrm{G} 1$ or S phase of the cell cycle, but not in G2/M (Supplemental Fig. S2A); there was a corresponding failure of tel2-1 cells to halt the cell cycle properly after DNA damage was inflicted in G1 or S, but not G2/M (Supplemental Fig. S2B-D). To abolish Rad53 phosphorylation, both MEC1 and TEL1 must be deleted. Strikingly, double mutant tel2-1 mec1s cells completely failed to phosphorylate Rad53 after DNA damage (Fig. 1B). In contrast, in tel1s tel2-1 cells, the phosphorylation of Rad53 after phleomycin treatment occurred to a similar extent, and at approximately the same rate, as in each of the single mutants. Hence, we conclude that tel2-1 disrupts the Tell pathway, rather than the Mecl pathway, of DNA damage signaling.

We next examined the stage in the Tell DNA damage response signaling pathway at which the tel2-1 mutation exerted its effect. Two proteins, Mrcl and Rad9, act in parallel pathways downstream from Mec1 and Tell to activate Rad53 (Fig. 1C; Alcasabas et al. 2001; Tanaka and Russell 2001). In tel2-1 cells, following DNA damage the phosphorylation of these two proteins was significantly delayed (Fig. 1D), demonstrating that Tel2 acts upstream of Rad9 and Mrc1.

Xrs2 is temporally one of the earliest proteins to localize to sites of DNA damage and is required for the TEL1-dependent response to DSBs (Wu et al. 2000; Zhao et al. 2000; Usui et al. 2001). Xrs2 is phosphorylated in a strictly TEL1-dependent manner after DNA damage and is a substrate for Tell in vitro (D'Amours and Jackson 2001; Usui et al. 2001; Mallory et al. 2003; Nakada et al. $2003 \mathrm{~b})$. We found that tel2-1 significantly delayed phosphorylation of Xrs2 after DNA damage (Fig. 1D). Taken together, all these results indicate that Tel2 acts as an upstream component of the TEL1 signaling pathway.

Mecl has been shown to associate constitutively with Ddc2, which is required for the localization of Mec1 to sites of DNA damage (Rouse and Jackson 2002). TEL2 and DDC2 encode proteins of similar size and share $24 \%$ identity at the primary sequence level (Supplemental Fig. S3). To test for structural similarity between these proteins, we used sequence-structure threading. This computational method uses both primary sequence and predicted secondary structure to compare a given protein against a database of known protein folds (see Materials and Methods). Strikingly, for both Tel2 and Ddc2, the highest-scored protein fold prediction identified the same protein: importin- $\beta$ (Supplemental Fig. S4A). These predictions were made with high confidence $(P$. value $<0.0001$ for Ddc2 and $P$-value $<0.001$ for Tel2). Importin- $\beta$ belongs to the ARM repeat superfamily of protein folds, as defined by the SCOP classification of protein structures (Murzin et al. 1995). This includes the HEAT repeat, Armadillo repeat, and other protein families, all of which adopt $\alpha$-superhelical three-dimensional structures. $\alpha$-Superhelices are found in proteins of diverse function, but many share the property of interaction with other $\alpha$-superhelices.

Tell and Mecl have been predicted previously to contain $\alpha$-superhelices in regions $\mathrm{N}$-terminal to their kinase domains (Perry and Kleckner 2003). Our independent analysis of Tell and Mec1 corroborated these predictions
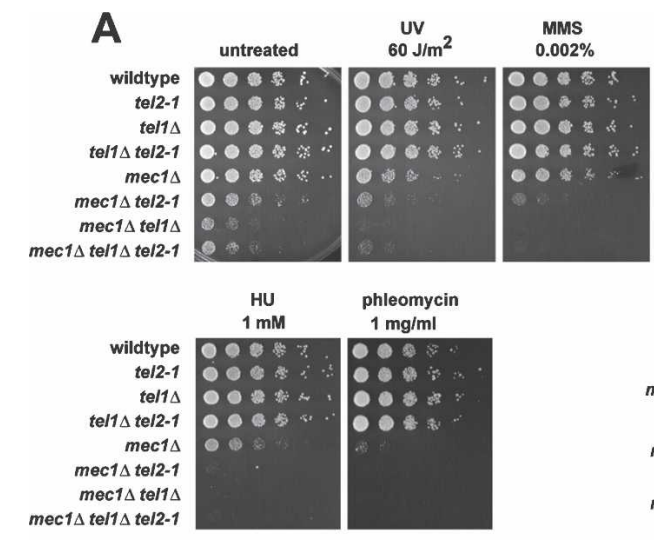
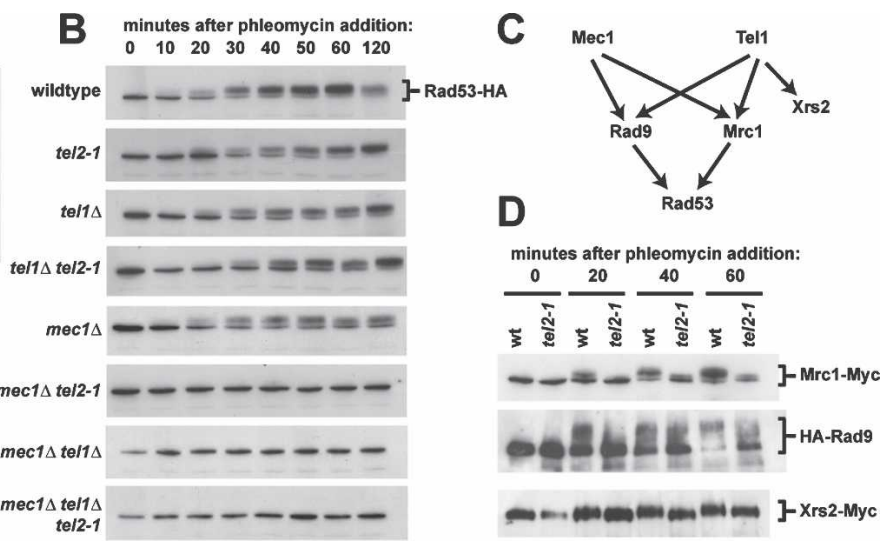

Figure 1. Tel2 is an upstream component of the TEL1 pathway of DNA damage signaling. Note that all mec1 $1 \Delta$ strains also contain a deletion of SML1. (A) Fivefold serial dilutions of logarithmically growing cells were plated onto media containing DNA-damaging drugs. For UV treatment, cells were irradiated at $254 \mathrm{~nm}$ immediately after plating. $(B) \alpha$-Factor-arrested cells were treated with $5 \mu \mathrm{g} / \mathrm{mL}$ phleomycin, and samples were collected every $10 \mathrm{~min}$ for Western blots. $(C)$ Diagram of DNA damage signaling pathways in $S$. cerevisiae. $(D) \alpha$-Factor-arrested cells were treated with $5 \mu \mathrm{g} / \mathrm{mL}$ phleomycin, and samples were collected every 20 min for Western blots. 
(Supplemental Fig. S4B). Specifically, the N-terminal part of each protein was predicted to consist of two ARM repeat $\alpha$-superhelices separated by another $\alpha$-superhelix composed of tetratricopeptide (TP) repeats; the C-terminal segment was predicted to contain a domain structurally similar to the PIK catalytic domain (Supplemental Fig. S4B). Based on the predictions of helical regions in the structures of Mec1 and Tel1, and because Ddc2 is known to interact with the $\mathrm{N}$-terminal $\alpha$-superhelical region of Mec1 (Wakayama et al. 2001), we predicted that Tel2 and Tell would also interact.

Indeed, epitope-tagged Tel2 and Tell expressed from their native promoters could be coimmunoprecipitated from whole-cell yeast extracts (Fig. 2A,B), and this interaction was mediated by the ARM/TP repeat region of Tel1 (Supplemental Figs. S5, S6). The interaction did not require the Tell kinase domain itself. The interaction detected by coimmunoprecipitation was unchanged by DNA damage (Fig. 2A,B). In contrast, Tel2-1 protein failed to coimmunoprecipitate with Tel1 (Fig. 2C), indicating that the tel2-1 point mutation disrupts the interaction of Tel2 with Tell. The expression levels of Tel2 and the mutant Tel2-1 were identical (Figs. 2C [input lanes], 3B). TEL2 is essential, yet cells expressing tel2-1 as their only copy of the TEL2 gene are alive and grow apparently normally (Fig. 1A), indicating that the Tel2-1 protein is not globally misfolded or degraded.
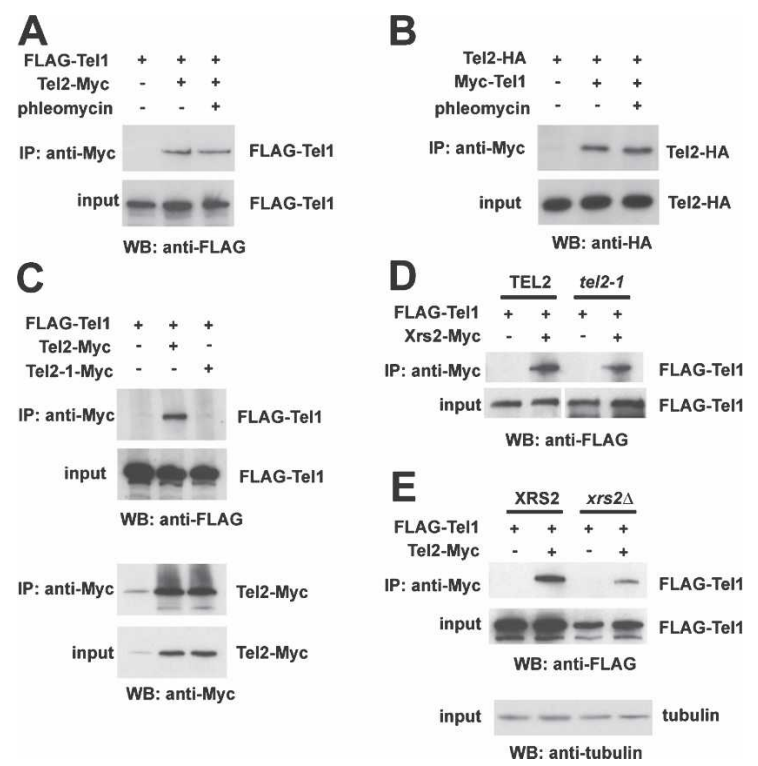

Figure 2. Interaction of Tell and Tel2. All proteins were expressed from their endogenous chromosomal loci as the only copy of the gene. $(A, B)$ Tell and Tel2 constitutively interact. In each experiment, half of the doubly tagged culture was treated with $5 \mu \mathrm{g} / \mathrm{mL}$ phleomycin for 30 min before collection of cells. (C) Tel2-1 fails to interact with Tell. Extracts were prepared from diploid strains TEL2/tel2-1, TEL2-Myc/tel2-1, and TEL2/tel2-1-Myc. (Top panels) Inputs and immunoprecipitations were subjected to anti-Flag immunoblotting. (Bottom panels) The same blots were stripped and reprobed with anti-Myc antibody. The faint band in the first lane is a result of Tel2-Myc protein bleeding over from the adjacent lane. (D) The Tel1-Xrs2 interaction does not depend on TEL2. More cells were used in the tel2-1 immunoprecipitations, in order to load approximately equal amounts of Flag-Tell protein in each immunoprecipitation. (E) The Tell-Tel2 interaction does not depend on $X R S 2$. Note that expression levels of Flag-Tel1 were lower in xrs2s strains.
Tel1 interacts with the Xrs2 subunit of the MRX complex, an interaction required for Tell localization to sites of DNA damage (Nakada et al. 2003a; Falck et al. 2005; You et al. 2005). However, this Tel1-Xrs2 interaction remained intact in tel2-1 cells (Fig. 2D). Furthermore, the interaction between Tell and Tel2 did not require $X R S 2$ (Fig. 2E). Finally, no interaction between Tel2 and Xrs2 was detected by coimmunoprecipitation (data not shown). We conclude that the Tel1-Tel2 interaction does not involve Xrs2.

We found that the tel2-1 mutation abrogated recruitment of Tel1 to a DSB. First, we found that tel2-1 did not alter nuclear localization of Tel1 (Fig. 3C), so the effects of the tel2-1 mutation cannot be explained by a failure of Tel1 to reach its DNA damage targets in the nucleus. We then performed chromatin immunoprecipitation (ChIP) using strains in which a single DSB could be induced by growth in galactose (Fig. 4A). Flag-Tell was specifically enriched at the break, as reported previously (Nakada et al. 2003a; Lisby et al. 2004). In tel2-1 cells, enrichment of Flag-Tel1 at the break dropped from $\sim 30$-fold to $\sim 7.5$-fold over the background signal from an untagged control strain (Fig. 4A). A recent study of Tel2 in mammalian cells reported that lack of Tel2 caused destabilization of ATM protein (Takai et al. 2007). The tel2-1 mutation, which we found disrupts the Tel1-Tel2 interaction, also led to somewhat lower Tell levels; however, in yeast, the drop in protein levels was less dramatic than reported in the mammalian situation, with Flag-Tell still being maintained at $\sim 60 \%-80 \%$ of its normal level (Fig. 3A). Loss of protein stability is commonly seen when a protein cannot interact with one of its usual partners; for example, we also observed lower Tell protein levels when XRS2 was deleted (Fig. 2E). We ruled out the possibility that reduced Tell protein levels might have accounted for the reduced association with the DSB. We expressed a second copy of Flag-Tel1 in tel2-1 cells from a plasmid, thereby boosting the Flag-Tell expression level to above the level found in a TEL2 strain (Fig. 4B). In these tel2-1 cells, Flag-Tel1 localization to a DSB was still defective (Fig. 4B) in spite of its overexpression. Hence, the tel2-1 mutation impairs Tell recruitment to a site of damage, and this impairment is not a result of lowered protein level of the PIKK Tell.

MRX binding to sites of damage precedes Tel1 localization, and the Tell-Xrs2 interaction is required for recruitment of Tell to a DNA break (Nakada et al. 2003a; Lisby et al. 2004; Falck et al. 2005; You et al. 2005). We found that Xrs2 localization to a DSB remained normal in tel2-1 cells (Fig. 4C). Hence, in tel2-1 cells, Tell remains competent to interact with Xrs2 (Fig. 2D) and Xrs2 binds normally to a DSB, yet Tell localization to a DSB is impaired.

We were unable to detect an interaction of Tel2 itself with a DNA break in vivo by ChIP or immunofluorescence in strains with an induced $\mathrm{HO}$ cut, or by immunofluorescence on meiotic chromosome spreads (data not shown). Therefore we propose that the Tel1-Tel2 complex substantially or completely dissociates prior to (or upon) binding of Tell to a DSB. This is consistent with experiments on purified human ATM, in which localization to dsDNA in vitro required only Mre11Rad50-Nbs1 (Lee and Paull 2005). Our results now suggest the possibility that the lack of added Tel2 in those experiments may help explain why that study did not recapitulate certain aspects of ATM activation in vivo. 
A

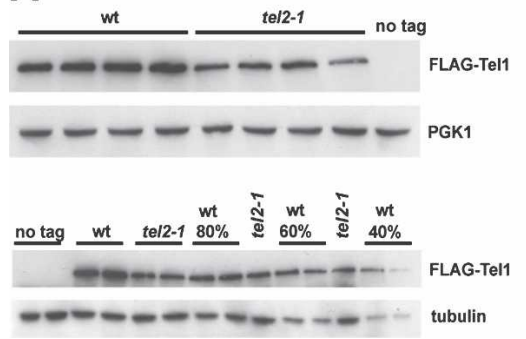

B

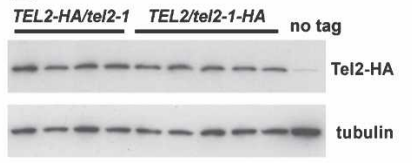

C

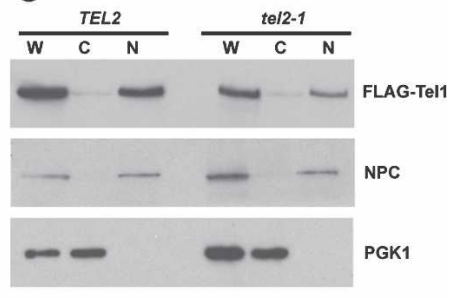

Figure 3. Effects of tel2-1 on Tell and Tel2 levels and distribution (A) Tell levels are lower in tel2-1 cells. (Top panel) Four isolates of each genotype (TEL2 or tel2-1) containing Flag-Tell were analyzed. Equal quantities (as measured by $\mathrm{OD}_{600}$ ) of logarithmically growing cells were collected, and whole-cell extracts were prepared by TCA precipitation and subjected to anti-Flag immunoblotting. The same samples were run on a separate gel, and the blot was probed with anti-PGK1 antibody as a loading control. (Bottom panel) TEL2 extract was diluted with SDS-PAGE loading buffer to various final concentrations (for example, "80\%" indicates the sample was diluted to $80 \%$ of its original concentration, which is a 4:5 dilution). Dilutions were run alongside undiluted tel2-1 extract and subjected to anti-Flag immunoblotting. The blot was stripped and reprobed with anti-tubulin antibody as a loading control. $(B)$ Tel2 protein levels are not altered by the tel2-1 mutation. Four diploid TEL2-HA/tel2-1 and five diploid TEL2/tel2-1-HA strains were analyzed. Logarithmically growing cells were collected and wholecell extracts were prepared by bead-beating in urea buffer. Equal amounts of total protein were loaded in each lane and subjected to anti-HA immunoblotting. The blot was stripped and reprobed with anti-tubulin antibody as a loading control. $(C)$ tel2-1 does not affect the nuclearcytoplasmic distribution of Tell. Logarithmically growing cells were homogenized to yield whole-cell extract (W), then separated into nuclear (N) and cytoplasmic $(\mathrm{C})$ fractions by differential centrifugation. Fractions were analyzed by immunoblotting with anti-Flag, anti-PGK1, or anti-nuclear pore complex (NPC) antibodies.

Our data show that the Tel1-Tel2 interaction regulates Tell localization to DSBs and its ability to activate its downstream targets. In an independent report on findings made during the same time as the research described in the present paper (Takai et al. 2007), it was suggested that the entire effect of mammalian Tel2 on the ATM pathway is due to destabilization of ATM protein. This decreased stability upon experimentally induced loss or mutation of Tel2 may reflect ATM's or Tel1's loss of normal interaction with its binding partner. While we find that loss of the yeast Tel1-Tel2 interaction leads to moderately lower Tell protein levels, our work clearly demonstrates that a lower Tell protein level cannot account for the effects of Tel2 on the Tell signaling pathway; rather, our results show that Tel2 is necessary for localization of Tell to a DSB even with full levels of Tell present.

The defects in tel2-1 cells were largely specific for the Tell pathway. Studies in $S$. pombe and mammalian cells have demonstrated a physical interaction between Tel2 and other PIKKs, a family that includes Tell/ATM and
Mec1/ATR (Collis et al. 2007; Hayashi et al. 2007; Takai et al. 2007). Our data do not rule out an interaction between Tel2 and Mec1 (or other PIKKs) in budding yeast. Indeed, we note that Rad53 phosphorylation after DNA damage was slightly delayed in tel1s tel2-1 cells compared with each single mutant, consistent with Tel2 having additional functions that do not lie upstream of Tel1. Such additional roles are also suggested by the fact that TEL2 is essential for viability, while TEL1 is not. However, the available data indicate that TEL2 is not essential due to an interaction with the essential MEC1 gene, because we found that sml1 $\Delta$, which rescues the lethality of mec1s, could not rescue the lethality of tel2s (data not shown). Therefore Tel2 is likely to have an essential function or functions unrelated to Mec1/ Tel1-dependent DNA damage signaling. Studies in other organisms suggest these other functions may include a role in DNA replication or survival of replication stress (Garcia-Muse and Boulton 2005; Collis et al. 2007; Shikata et al. 2007).

Our work reveals that both Tel2 and Ddc2 are struc-
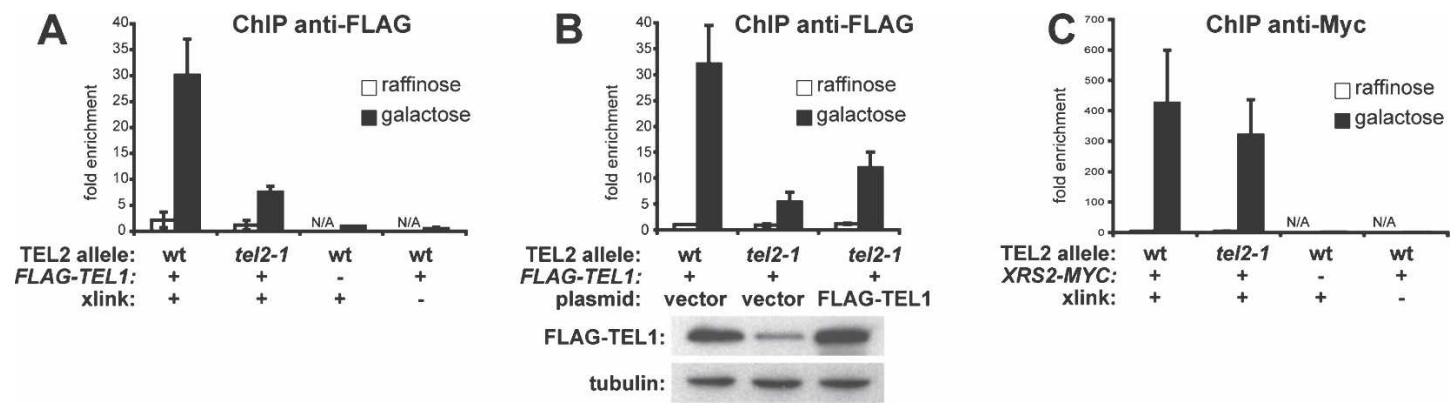

Figure 4. Tell localization to a DSB depends on its interaction with Tel2. A single DSB was induced in $\alpha$-factor-arrested cells by addition of $2 \%$ galactose for $1 \mathrm{~h}$. All strains contained a recognition site for HO endonuclease integrated at the TRP5 locus and a plasmid expressing HO endonuclease under the control of a galactose-inducible promoter; the endogenous HO recognition site at MATa was mutated to MATa-inc. DNA adjacent to the break site was detected by quantitative PCR. Data shown are the average of three experiments; error bars represent standard deviation of the mean. (A) Tell localization to a DSB is reduced in a tel2-1 strain. $(B)$ Reduced localization of Tell to a DSB is not a result of lower expression levels. An extra copy of Flag-Tell was expressed from the CEN plasmid pRS316 in tel2-1 cells, boosting the expression level above that seen in a TEL2 strain. (Bottom panel) Western blots of cells collected just before formaldehyde fixation of cultures for ChIP. (C) Xrs2 localization to a DSB is not affected by tel2-1. 
turally similar to importin- $\beta$, adopting a common $\alpha$-superhelical fold. Like Ddc2, Tel2 interacts with the ARM repeat-containing $\mathrm{N}$-terminal region of Tell. Therefore, this mode of protein-protein interaction via interactions between $\alpha$-superhelical regions may represent a general strategy by which various classes of proteins (including the PIKKs) can interact with their binding partners.

\section{Materials and methods}

Plasmids

Bacterial and yeast plasmids were obtained and constructed as described in the Supplemental Material.

Yeast strains

All strains were constructed in the S288C strain background and were isogenic with BY4736 (EHB13029) (Brachmann et al. 1998), as described in the Supplemental Material except as noted in Supplemental Table 1. The tel2-1 mutation was introduced into the S288C strain background by crossing AJL1291D (a gift from A. Lustig) to BY4736, then backcrossing to BY4736 at least five times. Rad53 was HA-tagged in wild-type and tel2-1 strains by integration of plasmid pEHB3212 (see the Supplemental Material). The MEC1, SML1, TEL1, and XRS2 ORFs were deleted by PCR-mediated gene disruption (Longtine et al. 1998). Tel2, Mrc1, Tel1, $\operatorname{Rad} 9$, and Xrs2 were tagged as described in the Supplemental Material. Haploid strains with an inducible DSB were made as described in the Supplemental Material.

\section{Damage sensitivity}

For analysis of damage sensitivity on plates, logarithmically growing cells were counted on a hemocytometer, and fivefold serial dilutions were prepared from a starting concentration of $4 \times 10^{6}$ cells per milliliter. After spotting on plates, some of the plates were irradiated at $254 \mathrm{~nm}$ in a Stratalinker (Stratagene) as indicated. Plates were photographed after incubation for $2 \mathrm{~d}$ at $30^{\circ} \mathrm{C}$.

Cell cycle experiments

All yeast cultures were grown at $30^{\circ} \mathrm{C}$ with shaking at $225 \mathrm{rpm}$. The G1, S-phase, and G2/M checkpoints and responses were tested using $\alpha$-factoror nocodazole-arrest protocols as described in the Supplemental Material. For flow cytometry, fixed cells were stained with SYBR Green I as described (Fortuna et al. 2001). Data were collected on a FACScalibur (BD Biosciences).

\section{Computational methods}

The alignment of Tel2 and Ddc2 (Supplemental Fig. S3) was obtained using the T-Coffee server (Notredame et al. 2000).

To structurally characterize Tel2, Ddc2, Tel1, and Mec1 (Supplemental Fig. S4), we determined their domain architectures and a three-dimensional fold of each domain using the PSIPRED protein structure prediction server (McGuffin et al. 2000). Specifically, the folds were assigned by threading the whole sequence through a library of all known folds, relying on similarities in sequence, predicted secondary structure, and statistical potentials for residue accessibilities and residue-residue distances as implemented in GenTHREADER (McGuffin and Jones 2003). For each predicted fold, a $P$-value was also calculated that estimates the probability of a false prediction, and a confidence level was assigned that relates to the $P$-value within a particular range. The current implementation of GenTHREADER requires that the target sequence is shorter than 1000 residues. When the target was longer, it was divided into segments of 1000 residues that overlapped by 300 residues, each of which was independently processed by PSIPRED. Next, the top-scoring nonoverlapping folds that maximized the coverage of the entire sequence were selected. The fold families were assigned using the structural classification of protein domains, SCOP (Murzin et al. 1995). All folds were predicted at the highest confidence level (CERTAIN, $P$-value $<0.0001$ ), except for Tel2 and the first domain of Tell, which were predicted at the second-highest level (HIGH, $P$-value $<0.001)$. For both Tel2 and Ddc2, the highest-scored protein fold prediction identified the same protein, importin- $\beta$ (PDB ID: 2 BKU, chain B). The two closest structures for the first ARM repeats of Tell and Mecl were importin- $\beta$ structures solved independently (PDB IDs: 2BKU, chain B and 1QGR, respectively). The closest known structures to the TP repeat domain (PDB ID: 1W3B) as well as to the second ARM repeat together with PI3K catalytic domain (PDB ID for both: 1E8X) were the same for Tell and Mec1.

\section{Western blotting}

For detection of Rad53-HA and Flag-Tell, protein was prepared by TCA precipitation. For detection of Mrc1-Myc, Xrs2-Myc, HA-Rad9, Tel2Myc, and Tel2-HA, protein was prepared by bead-beating in urea buffer. Detailed protein preparation, electrophoresis, and blotting conditions are described in the Supplemental Material.

\section{Coimmunoprecipitation}

Whole-cell extracts were prepared by bead-beating in lysis buffer and were subjected to immunoprecipitation as described in the Supplemental Material.

\section{Nuclear-cytoplasmic fractionation}

Cells were disrupted by Dounce homogenization and fractionated by differential centrifugation. Details are given in the Supplemental Material.

\section{ChIP}

ChIP was performed essentially as described (Taggart et al. 2002), with differences as described in the Supplemental Material. DNA was detected by quantitative real-time PCR. Fold enrichment of the HO cut was calculated as $\left[\left(\mathrm{IP}_{\mathrm{HO}}\right) /\right.$ input $\left.\left._{\mathrm{HO}}\right)\right]$, with the enrichment of the untagged control strain set to 1 . Note that for the untagged and uncross-linked controls, only galactose-induced cultures were analyzed. As a reference, the same samples were also amplified using a different primer set (ARO). Signals from the ARO primers are shown in Supplemental Figure S7.

\section{Acknowledgments}

We thank S. Nautiyal and A. Lustig for yeast strains; D. Toczyski, J. Li, D. Faucher, and R. Wellinger for plasmids and reagents; M. Kampmann for technical advice; and D. Morgan, D. Toczyski, and J. Li for helpful discussions and critical reading of the manuscript. This work was supported by an NIH grant to E.H.B. and an NSF Graduate Research Fellowship to C.M.A.

\section{References}

Abraham, R.T. 2001. Cell cycle checkpoint signaling through the ATM and ATR kinases. Genes \& Dev. 15: 2177-2196.

Ahmed, S., Alpi, A., Hengartner, M.O., and Gartner, A. 2001. C. elegans RAD-5/CLK-2 defines a new DNA damage checkpoint protein. Curr. Biol. 11: 1934-1944.

Alcasabas, A.A., Osborn, A.J., Bachant, J., Hu, F., Werler, P.J., Bousset, K., Furuya, K., Diffley, J.F., Carr, A.M., and Elledge, S.J. 2001. Mrc1 transduces signals of DNA replication stress to activate Rad53. Nat. Cell Biol. 3: 958-965.

Brachmann, C.B., Davies, A., Cost, G.J., Caputo, E., Li, J., Hieter, P., and Boeke, J.D. 1998. Designer deletion strains derived from Saccharomyces cerevisiae S288C: A useful set of strains and plasmids for PCR-mediated gene disruption and other applications. Yeast 14: 115132.

Collis, S.J., Barber, L.J., Clark, A.J., Martin, J.S., Ward, J.D., and Boulton, S.J. 2007. HCLK2 is essential for the mammalian S-phase checkpoint and impacts on Chk1 stability. Nat. Cell Biol. 9: 391-401.

D'Amours, D. and Jackson, S.P. 2001. The yeast Xrs2 complex functions in S phase checkpoint regulation. Genes \& Dev. 15: 2238-2249.

Falck, J., Coates, J., and Jackson, S.P. 2005. Conserved modes of recruitment of ATM, ATR and DNA-PKcs to sites of DNA damage. Nature 434: 605-611.

Fortuna, M., Sousa, M.J., Côrte-Real, M., Leão, C., Salvador, A., and Sansonetty, F. 2001. Cell cycle analysis of yeasts. In Current protocols in cytometry (ed. J.P. Robinson et al.), Unit 11.13. John Wiley \& Sons, New York. doi: 10.1002/0471142956.cy1113s13.

Garcia-Muse, T. and Boulton, S.J. 2005. Distinct modes of ATR activation after replication stress and DNA double-strand breaks in Caenorhabditis elegans. EMBO J. 24: 4345-4355.

Gartner, A., Milstein, S., Ahmed, S., Hodgkin, J., and Hengartner, M.O. 2000. A conserved checkpoint pathway mediates DNA damage-induced apoptosis and cell cycle arrest in C. elegans. Mol. Cell 5: 435443. 
Hartman, P.S. and Herman, R.K. 1982. Radiation-sensitive mutants of Caenorhabditis elegans. Genetics 102: 159-178.

Hayashi, T., Hatanaka, M., Nagao, K., Nakaseko, Y., Kanoh, J., Kokubu, A., Ebe, M., and Yanagida, M. 2007. Rapamycin sensitivity of the Schizosaccharomyces pombe tor2 mutant and organization of two highly phosphorylated TOR complexes by specific and common subunits. Genes Cells 12: 1357-1370.

Lee, J.H. and Paull, T.T. 2005. ATM activation by DNA double-strand breaks through the Mrel1-Rad50-Nbs1 complex. Science 308: 551554 .

Lisby, M., Barlow, J.H., Burgess, R.C., and Rothstein, R. 2004. Choreography of the DNA damage response: Spatiotemporal relationships among checkpoint and repair proteins. Cell 118: 699-713.

Longtine, M.S., McKenzie III, A., Demarini, D.J., Shah, N.G., Wach, A., Brachat, A., Philippsen, P., and Pringle, J.R. 1998. Additional modules for versatile and economical PCR-based gene deletion and modification in Saccharomyces cerevisiae. Yeast 14: 953-961.

Mallory, J.C., Bashkirov, V.I., Trujillo, K.M., Solinger, J.A., Dominska, M., Sung, P., Heyer, W.D., and Petes, T.D. 2003. Amino acid changes in Xrs2p, Dunlp, and Rfa2p that remove the preferred targets of the ATM family of protein kinases do not affect DNA repair or telomere length in Saccharomyces cerevisiae. DNA Repair (Amst.) 2: 10411064.

McGuffin, L.J. and Jones, D.T. 2003. Improvement of the GenTHREADER method for genomic fold recognition. Bioinformatics 19: $874-881$.

McGuffin, L.J., Bryson, K., and Jones, D.T. 2000. The PSIPRED protein structure prediction server. Bioinformatics 16: 404-405.

Morrow, D.M., Tagle, D.A., Shiloh, Y., Collins, F.S., and Hieter, P. 1995. TEL1, an S. cerevisiae homolog of the human gene mutated in ataxia telangiectasia, is functionally related to the yeast checkpoint gene MEC1. Cell 82: 831-840.

Murzin, A.G., Brenner, S.E., Hubbard, T., and Chothia, C. 1995. SCOP: A structural classification of proteins database for the investigation of sequences and structures. J. Mol. Biol. 247: 536-540.

Nakada, D., Matsumoto, K., and Sugimoto, K. 2003a. ATM-related Tell associates with double-strand breaks through an Xrs2-dependent mechanism. Genes \& Dev. 17: 1957-1962.

Nakada, D., Shimomura, T., Matsumoto, K., and Sugimoto, K. 2003b. The ATM-related Tell protein of Saccharomyces cerevisiae controls a checkpoint response following phleomycin treatment. Nucleic Acids Res. 31: 1715-1724.

Notredame, C., Higgins, D.G., and Heringa, J. 2000. T-Coffee: A novel method for fast and accurate multiple sequence alignment. J. Mol. Biol. 302: 205-217.

Perry, J. and Kleckner, N. 2003. The ATRs, ATMs, and TORs are giant HEAT repeat proteins. Cell 112: 151-155.

Rouse, J. and Jackson, S.P. 2002. Lcdlp recruits Meclp to DNA lesions in vitro and in vivo. Mol. Cell 9: 857-869.

Runge, K.W. and Zakian, V.A. 1996. TEL2, an essential gene required for telomere length regulation and telomere position effect in Saccharomyces cerevisiae. Mol. Cell. Biol. 16: 3094-3105.

Sanchez, Y., Desany, B.A., Jones, W.J., Liu, Q., Wang, B., and Elledge, S.J. 1996. Regulation of RAD53 by the ATM-like kinases MEC1 and TEL1 in yeast cell cycle checkpoint pathways. Science 271: 357-360.

Shikata, M., Ishikawa, F., and Kanoh, J. 2007. Tel2 is required for activation of the Mrcl-mediated replication checkpoint. J. Biol. Chem. 282: $5346-5355$.

Taggart, A.K., Teng, S.C., and Zakian, V.A. 2002. Estlp as a cell cycleregulated activator of telomere-bound telomerase. Science 297: 10231026.

Takai, H., Wang, R.C., Takai, K.K., Yang, H., and de Lange, T. 2007. Tel2 regulates the stability of PI3K-related protein kinases. Cell 131: 1248-1259.

Tanaka, K. and Russell, P. 2001. Mrc1 channels the DNA replication arrest signal to checkpoint kinase Cds1. Nat. Cell Biol. 3: 966-972.

Usui, T., Ogawa, H., and Petrini, J.H. 2001. A DNA damage response pathway controlled by Tell and the Mrell complex. Mol. Cell 7: $1255-1266$.

Wakayama, T., Kondo, T., Ando, S., Matsumoto, K., and Sugimoto, K. 2001. Piel, a protein interacting with Mecl, controls cell growth and checkpoint responses in Saccharomyces cerevisiae. Mol. Cell. Biol. 21: 755-764.
Wu, X., Ranganathan, V., Weisman, D.S., Heine, W.F., Ciccone, D.N., O'Neill, T.B., Crick, K.E., Pierce, K.A., Lane, W.S., Rathbun, G., et al. 2000. ATM phosphorylation of Nijmegen breakage syndrome protein is required in a DNA damage response. Nature 405: 477-482.

You, Z., Chahwan, C., Bailis, J., Hunter, T., and Russell, P. 2005. ATM activation and its recruitment to damaged DNA require binding to the C terminus of Nbs1. Mol. Cell. Biol. 25: 5363-5379.

Zhao, S., Weng, Y.C., Yuan, S.S., Lin, Y.T., Hsu, H.C., Lin, S.C., Gerbino, E., Song, M.H., Zdzienicka, M.Z., Gatti, R.A., et al. 2000. Functional link between ataxia-telangiectasia and Nijmegen breakage syndrome gene products. Nature 405: 473-477.

Zou, L. and Elledge, S.J. 2003. Sensing DNA damage through ATRIP recognition of RPA-ssDNA complexes. Science 300: 1542-1548. 


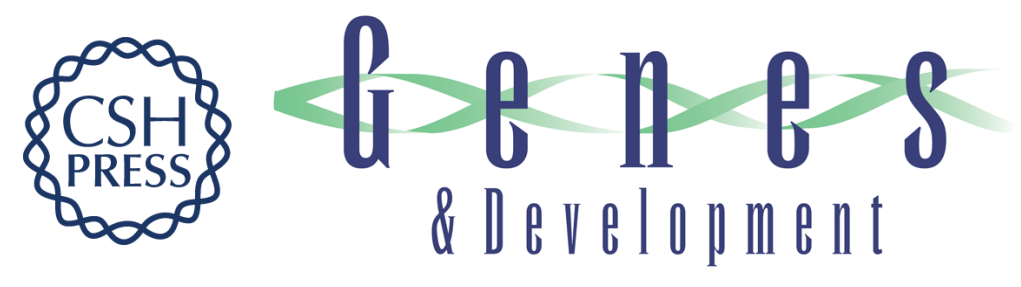

\section{Tel2 mediates activation and localization of ATM/Tel1 kinase to a double-strand break}

Carol M. Anderson, Dmitry Korkin, Dana L. Smith, et al.

Genes Dev. 2008, 22: originally published online March 11, 2008

Access the most recent version at doi:10.1101/gad.1646208

\section{Supplemental http://genesdev.cshlp.org/content/suppl/2008/03/11/gad.1646208.DC1 Material}

References This article cites 36 articles, 13 of which can be accessed free at: http://genesdev.cshlp.org/content/22/7/854.full.html\#ref-list-1

License Freely available online through the Genes \& Development Open Access option.
Email Alerting Service

